\title{
EFECTO DEL TIPO Y CONCENTRACIÓN DE AGLUTINANTE EN LA CONDUCTIVIDAD TÉRMICA DE POLVOS DE CARBÓN
}

\section{THE EFFECT OF AGGLUTINANT CONCENTRATION AND TYPE ON COAL-POWDER THERMAL CONDUCTIVITY}

\begin{abstract}
Jorge Hernando Bautista Ruiz
Licenciado en Física y Matemáticas, Magister en Metalurgia y Ciencia de los Materiales, Profesor

Auxiliar, Universidad Francisco de Paula Santander, Cúcuta, Colombia.

jorgebautista@ufps.edu.co

Gabriel Peña Rodríguez

Licenciado en Física, Magister en Física, Doctorado en Ingeniería de los Materiales.

Profesor Asociado, Universidad Francisco de Paula Santander, Cúcuta, Colombia.

ggabrielp@yahoo.com

William Aperador Chaparro

Físico, Magister en Metalurgia y Ciencia de los Materiales, Doctor en Ingeniería de los Materiales

Profesor Asistente, Universidad Militar Nueva Granada, Bogotá, Colombia.

william.aperador@unimilitar.edu.co
\end{abstract}

Fecha de recepción: 17 de enero de 2011

Fecha de aprobación: 11 de mayo de 2011

\section{RESUMEN}

Se reporta la conductividad térmica (k) a temperatura ambiente de polvos de carbón térmico, en función del tipo y concentración del aglutinante (agua, alcohol, ACPM), los cuales variaron de porcentaje en peso (\%wt), entre 5\% y $40 \%$. Las muestras fueron obtenidas de la mina Cerro Tasajero ubicada en el área metropolitana de San José de Cúcuta, Colombia, las cuales se pulverizaron manualmente, utilizando molino de rodillos y se tamizaron en malla pasante número $100(150 \mu \mathrm{m})$. $\boldsymbol{k}$ se determinó, usando el principio físico de flujo lineal transitorio de calor, mediante el sistema KD2 ${ }^{\circledR}$ Thermal Properties Analyzer (Decagon Devices, Inc). El porcentaje de ceniza presente en la muestra, se determinó según la norma ASTM D3174, y se encontró un valor de 1,75\%; para hallar la concentración de azufre, se utilizó la norma ASTM D3177, y reportó un valor de $0,45 \%$, mientras que el porcentaje de materia volátil presente en la muestra fue de $34,17 \%$, que se halló, aplicando la norma ASTM D3175. El análisis de $\boldsymbol{k}$ en función del tipo y concentración del aglutinante, se realizó, usando el ajuste a los datos experimentales mediante la función logística ${ }^{y=\frac{\left(A_{1}-A_{2}\right)}{\left[1-\left(x / x_{0}\right)^{n}\right]}+A_{2}}$ donde $A_{2}$ corresponde a los valores de la propiedad térmica $(y)$ para la concentración inicial de aglutinante $(x) ; A_{1}$ representa dicho valor para cuando la concentración de aglutinante $(x)$ sea muy grande; $x_{0}$ corresponde al punto donde sucede el cambio de curvatura y $n$ la razón de crecimiento $o$ 
decrecimiento. Los resultados encontrados en este trabajo, podrán ser utilizados en el desarrollo de estudios que permitan la fabricación de cilindros de polvos de carbón prensados a altas presiones, los cuales se usarán en los procesos de combustión en hornos industriales que se utilizan en la sinterización de productos cerámicos como teja, bloque y baldosa.

Palabras clave: carbón térmico, conductividad térmica, flujo de calor lineal transitorio.

\section{ABSTRACT}

This paper reports a thermal conductivity (k) given an environment temperature of thermal coal powder as a function of agglutinant concentration and type -i.e. water, alcohol, diesel- which changed their weight percentage (\%wt) between 5-and-40 percent. Samples were obtained from Cerro Tasajero, a mine located at San Jose de Cucuta metropolitan area, which were pulverized manually using roller mill and sifted by a mesh No. $100(150 \mu \mathrm{m})$. The $\boldsymbol{k}$ value was determined using the physical principle of temporary linear heat flow by system KD2 ${ }^{\square}$ Thermal Properties Analyzer (Decagon Devices, Inc.) The ash percentage in the sample was given by norm ASTM D 3174, providing a value of 1.75 percent. The sulfur concentration was obtained using norm ASTM 3177, reporting a value of 0.45 percent, while the percentage of matter volatile in the sample was 34.17 percent by applying norm ASTM 3175. The analysis of $\boldsymbol{k}$ as a function of agglutinant type and concentration was carried out adjusting the experimental data based on the logistical function $y=\frac{\left(A_{1}-A_{2}\right)}{\left[1-\left(x / x_{0}\right)^{n}\right]}+A_{2}$ where $A_{2}$ corresponds to values of the thermal property $(y)$ for an initial concentration of agglutinant $(x) . A_{1}$ represents such a value as agglutinant $(x)$ concentration may be very big; $x_{0}$ reveals the point where bend is changing and $n$ the reason of growth or falling. The results in this experience could be used to develop other studies in order to produce high-pressed coal powder cylinders, which will be used in industrial burning processes by sintering ceramic goods, such as roof tile, block and wall tile.

Key words: thermal coal, thermal conductivity, transient linear heat flow.

\section{INTRODUCCIÓN}

El carbón es el combustible fósil más abundante, no sólo porque existen grandes reservas, sino que éstas se encuentran repartidas abundantemente en el Globo. Actualmente, el carbón es la mayor fuente de energía primaria utilizada por las termoeléctricas, y la industria siderúrgica, así como fuente de combustión en los 
procesos de quema o cocción de productos cerámicos tradicionales como ladrillos, tejas y baldosas [1], [2], [3].

El bajo costo y la disponibilidad del carbón, permitirá por muchos años más su uso como combustible o fuente de energía, especialmente en los países en vía de desarrollo.

Los problemas medioambientales derivados del uso de combustibles fósiles han impulsado el desarrollo de tecnologías encaminadas a mejorar la eficiencia y reducir las emisiones contaminantes a los cada vez más restrictivos niveles adoptados en los protocolos internacionales [4]. El carbón tiene muchos usos importantes, aunque los más significativos son la producción de electricidad, la fabricación de acero, cemento y otros procesos industriales de producción de calor [5], [6].

En los últimos años, Colombia ha aumentado su participación en las exportaciones de carbón térmico y carbón coquizable, con una buena contribución de la zona de Norte de Santander [7], [8]. Es indispensable desarrollar estudios que contribuyan a mejorar las condiciones para la explotación y utilización del carbón, de manera que sea posible disminuir pérdidas y lograr el máximo rendimiento en su utilización y cumplir con las normas medioambientales.

Los resultados encontrados en el desarrollo de esta investigación, serán utilizados con el objetivo de fabricar cilindros de carbón compactados a altas presiones, los cuales se usarán en los procesos de combustión de hornos en la industria de producción de cerámicas que son relevantes en el área metropolitana de San José de Cúcuta y su área fronteriza con la República Bolivariana de Venezuela.

\section{PROCEDIMIENTO EXPERIMENTAL}

Preparación de las muestras. Se tomaron muestras representativas de carbón mineral de una de las minas más importantes de la región, la mina Cerro Tasajero, ubicada en el área metropolitana de San José de Cúcuta. Las muestras se pulverizaron manualmente en molino de rodillos. El polvo obtenido se tamizó en malla pasante número $100(150 \mu \mathrm{m})$. Utilizando el secadero de circulación forzada marca Gabrielli ${ }^{\circledR}$, a una temperatura de $312,0 \pm 0,5 \mathrm{~K}$ durante 24 horas, se realizó el proceso de secado; a la masa inicial de la muestra, se le agregaron tres tipos diferentes de aglutinantes: agua destilada, alcohol comercial al $96 \%$ y ACPM en porcentaje en peso con variaciones cada $5 \%$ en un rango comprendido entre el $5 \%$ y $40 \%$. Luego se compactó con una carga constante de $5315,6 \pm 0,2$ gf. Posteriormente, se procedió a medir la conductividad térmica (k) con el sistema KD2-Pro $(R)$, tomando cinco lecturas de $\boldsymbol{k}$, con una diferencia entre medidas de cinco minutos y después se promediaron. Para complementar la caracterización de 
las muestras de carbón de la mina Cerro Tasajero, el porcentaje de ceniza se determinó usando la norma ASTM D3174; la concentración de azufre se halló, empleando la norma ASTM D3177, mientras que la materia volátil se obtuvo, según la norma ASTM D3175.

Método experimental. El sistema KD2-Pro ${ }^{\circledR}$, permite hallar las propiedades térmicas, usando como principio físico del flujo lineal transitorio de calor, en donde se monitorea el perfil de temperatura en función del tiempo. El sistema realiza mediciones experimentales que se ajustan a funciones exponenciales integrales mediante un procedimiento no lineal de mínimos cuadrados. Un término de la tendencia lineal, corrige los cambios de temperatura de la muestra durante la medida, optimizando así la exactitud de las lecturas [9].

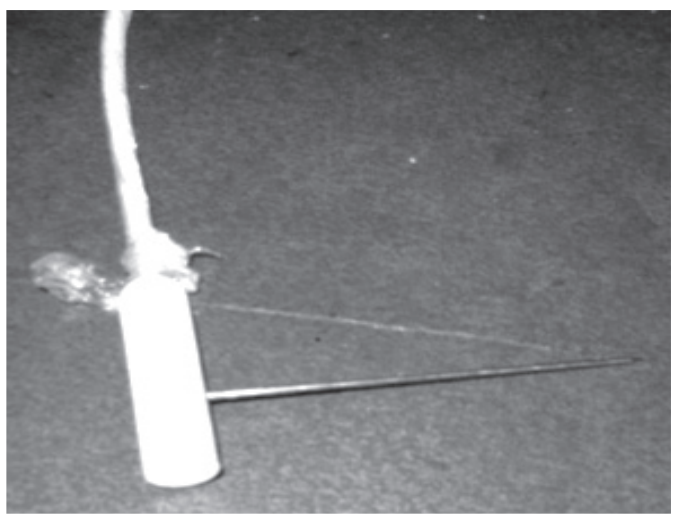

(a)

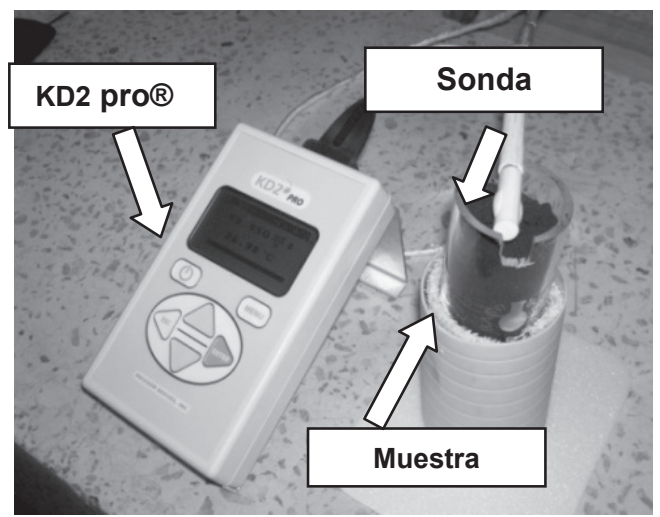

(b)

Figura 1. Sistema KD2-Pro®. (a) sensor o sonda. (b) montaje experimental

La figura 1(a), muestra el sistema KD2-Pro(R ) junto con la sonda o aguja empleada en el desarrollo del presente trabajo. En la figura 1(b), se presenta el diseño experimental empleado para efectuar las mediciones de las propiedades térmicas del polvo de carbón compactado con diferentes porcentajes en peso del aglutinante. Se visualiza la ubicación de la sonda o sensor, sobre la muestra de carbón y su conexión con el analizador KD2-Pro(R ).

Los valores de $k$ se determinan con el monitoreo de la disipación del calor que proviene de una fuente lineal transitoria dada por un voltaje conocido. El gradiente de temperatura para un medio infinito, a una distancia $r(m)$ próxima a la fuente lineal de calor, está dado por Carslaw H. S., et al [10]. 


$$
\Delta T=\frac{Q}{4 \pi k t} e^{\left(-\frac{r^{2}}{4 D t}\right)}
$$

Donde $k$ es la conductividad térmica $\left(\mathrm{W} \mathrm{m}^{-1} \mathrm{~K}^{-1}\right), D$ es la difusividad térmica $\left(\mathrm{m}^{2} \mathrm{~s}^{-1}\right)$, $\mathrm{Q}\left(\mathrm{J} \mathrm{m}^{-1}\right)$ corresponde a la cantidad de calor aplicada en forma instantánea, y $t(\mathrm{~s})$ es tiempo. Considerando a $Q$ como un pulso instantáneo de calor en un intervalo de tiempo $\left(0<t<t_{1}\right)$, la ecuación (1) se transforma en:

$$
\Delta T=-\frac{q}{4 \pi k} E i\left(-\frac{r^{2}}{4 D t}\right) \quad 0<t<t_{1}
$$

Donde $q$ es la tasa de disipación de calor $(\mathrm{W} / \mathrm{m}), t_{1}$ es el tiempo de calentamiento, y $E i$ es la integral exponencial [11]. El gradiente de temperatura después de interrumpir el pulso de calor en el proceso de enfreamiento ( $\mathrm{t}>\mathrm{t} 1)$ está dado por la ecuación 3 de Abramowitz [11]:

$$
\Delta T=-\frac{q}{4 \pi k}\left[-E i\left(-\frac{r^{2}}{4 D t}\right)+E i\left(-\frac{r^{2}}{4 D\left(t-t_{1}\right)}\right)\right] \quad t>t_{1}
$$

La conductividad térmica $(\boldsymbol{k})$ de la muestra en estudio, se determina ajustando los datos de temperatura tomados durante el tiempo de calentamiento, ecuación (2), y durante el enfriamiento, ecuación (3). Dicho ajuste es no-lineal de mínimos cuadrados [12], en donde los valores de $\boldsymbol{k}$ se minimizan con la diferencia entre el modelado y el medido por el sensor de temperatura. Un factor de tendencia lineal se incluye en el procedimiento inverso. La mayoría de los experimentos no ocurre bajo condiciones constantes de temperatura, por tal razón, se asume que la temperatura puede ser de tendencia lineal y se utiliza un parámetro de ajuste a esta tendencia, permitiendo reducir el error en forma sustancial.

Otros estudios teórico para hallar $\boldsymbol{k}$, donde se considera la fuente pulsada de forma cilíndrica de radio $a(\mathrm{~m})$ y longitud $2 b(\mathrm{~m})$, midiendo la temperatura en el centro de la muestra, reporta que el gradiente de temperatura durante el calentamiento $(0<t<$ $\left.t_{1}\right)$, es dado por la ecuación (4), y durante el enfriamiento $\left(\mathrm{t}>\mathrm{t}_{1}\right)$, por la expresión (5) [13].

$$
\begin{aligned}
& \Delta T=\frac{q}{4 \pi k} \int_{r^{2} / 4 D t}^{\infty} u^{-1} \exp (-u) \exp \left[-(a / r)^{2} u\right] I_{0}(2 a u / r) \operatorname{erf}\left(\frac{b}{r} \sqrt{u}\right) d u \\
& \Delta T=\frac{q}{4 \pi k} \int_{r^{2} / 4 D t}^{r^{2} / 4 D\left(t-t_{1}\right)} u^{-1} \exp (-u) \exp \left[-(a / r)^{2} u\right] I_{0}(2 a u / r) \operatorname{erf}\left(\frac{b}{r} \sqrt{u}\right) d u
\end{aligned}
$$

Donde, $I_{0}(x)$ representa una función de Bessel modificada de orden cero, $\operatorname{erf}(x)$ es la función error, y $u$ es una variable de integración. 
Si $a / r$ se aproxima a cero, y $b / r$ a infinito, se reducen las ecuaciones (4) y (5) a las expresiones (2) y (3).

Para el tipo de sensor y microcontrolador que maneja el sistema KD2, se ha encontrado que las ecuaciones (2) y (3), ajustan los datos del gradiente de temperatura tan bien como las ecuaciones (5) y (6), pero dan valores un poco diferentes para el ajuste en los parámetros, variación que debe incluirse en la calibración del equipo, así las ecuaciones más simples (2) y (3) son confiables para determinar el valor de $\boldsymbol{k}$.

\section{RESULTADOS Y DISCUSIÓN}

Una vez obtenidas las muestras, se procedió a analizar el porcentaje de ceniza, materia volátil, carbón fijo, azufre y el poder calorífico. Las normas ASTM D [14] se utilizaron en la determinación de los parámetros anteriores que se encuentran registrados en la tabla 1.

De los resultados anteriores y usando la norma ASTM D 388-82, que clasifica el carbón según el contenido de carbón fijo y materia volátil, es posible afirmar que las muestras de carbón de la mina Cerro Tasajero corresponden a un carbón tipo bituminoso clasificado como alto volátil (C.F $<69 \%$ y $M . V>31 \%$ ), que puede ser utilizado como carbón aglomerante. Este resultado implica la existencia de un alto potencial como materia prima para fabricar briquetas sólidas compactadas.

Tabla 1. Porcentaje en peso de ceniza, materia volátil, carbono, azufre y el poder calorífico.

\begin{tabular}{|l|c|}
\hline Análisis & Porcentaje (\%) \\
\hline Ceniza & 1,75 \\
\hline Material volátil (M.V) & 34,17 \\
\hline Carbón Fijo (C.F) & 64,08 \\
\hline Azufre & 0,45 \\
\hline Poder Calorífico (P.C) & $8450 \mathrm{Cal} / \mathrm{g}$ \\
\hline
\end{tabular}

En las tablas 2, 3 y 4 se presentan los resultados experimentales encontrados de la densidad $(\rho)$, conductividad térmica promedio $(k)$ y temperatura $(T)$ para las muestras de polvo de carbón de la mina Cerro Tasajero, en función de la concentración de fluido (agua, alcohol y ACPM), respectivamente, y para la presión de compactación constante de $52 \mathrm{~N}$. De los resultados anteriores, se aprecia que el valor promedio de $k$, para la muestra de carbón en polvo con tamaño de grano inferior a $150 \mu \mathrm{m}$ sin fluido $(0 \%)$, reporta un valor promedio de $(0,063 \pm 0,003) \mathrm{W} \mathrm{m}^{-1}$ $\mathrm{K}^{-1}$ para una temperatura promedio de $(300,85 \pm 1,02) \mathrm{K}$, valor que es similar al reportado $\left(0,065 \mathrm{~W} \mathrm{~m}^{-1} \mathrm{~K}^{-1}\right)$ por $\mathrm{Y} . \mathrm{S}$. Toulokian et al [15]. 
Tabla 2. Densidad, conductividad térmica y temperatura para el carbón en polvo con agua.

\begin{tabular}{|c|c|c|c|}
\hline \% AGUA & $\boldsymbol{\rho}\left(\mathbf{K g} \mathbf{m}^{\mathbf{3}}\right)$ & $\boldsymbol{k} \mathbf{( W m}^{-\mathbf{1}} \mathbf{K}^{-\mathbf{1}} \mathbf{)}$ & $\boldsymbol{T} \mathbf{( K )}$ \\
\hline 0 & $733,3 \pm 3,7$ & $0,062 \pm 0,004$ & $300,71 \pm 0,34$ \\
\hline 5 & $752,2 \pm 1,5$ & $0,112 \pm 0,004$ & $299,77 \pm 0,16$ \\
\hline 10 & $769,5 \pm 1,6$ & $0,134 \pm 0,003$ & $302,62 \pm 0,25$ \\
\hline 15 & $778,2 \pm 1,9$ & $0,184 \pm 0,005$ & $302,68 \pm 0,17$ \\
\hline 20 & $794,6 \pm 2,0$ & $0,193 \pm 0,005$ & $300,02 \pm 0,20$ \\
\hline 25 & $761,8 \pm 1,5$ & $0,221 \pm 0,004$ & $302,91 \pm 0,16$ \\
\hline 30 & $797,2 \pm 1,9$ & $0,238 \pm 0,005$ & $300,74 \pm 1,14$ \\
\hline 35 & $816,2 \pm 1,8$ & $0,261 \pm 0,003$ & $299,97 \pm 0,16$ \\
\hline 40 & $926,2 \pm 2,1$ & $0,277 \pm 0,003$ & $300,49 \pm 0,02$ \\
\hline
\end{tabular}

Tabla 3. Densidad, conductividad térmica y temperatura para el carbón en polvo con alcohol.

\begin{tabular}{|c|c|c|c|}
\hline$\%$ ALCOHOL & $\left.\boldsymbol{\rho}\left(\mathbf{K g} / \mathbf{m}^{3}\right)\right)$ & $\boldsymbol{k}\left(\mathbf{W m}^{-1} \mathbf{K}^{-1}\right)$ & $\boldsymbol{T}(\mathbf{K})$ \\
\hline 0 & $664,2 \pm 1,3$ & $0,063 \pm 0,002$ & $301,75 \pm 0,09$ \\
\hline 5 & $661,3 \pm 1,3$ & $0,101 \pm 0,004$ & $299,18 \pm 0,23$ \\
\hline 10 & $654,6 \pm 1,6$ & $0,118 \pm 0,003$ & $297,57 \pm 0,37$ \\
\hline 15 & $649,5 \pm 1,6$ & $0,120 \pm 0,001$ & $300,01 \pm 0,36$ \\
\hline 20 & $648,2 \pm 1,5$ & $0,134 \pm 0,002$ & $301,39 \pm 0,36$ \\
\hline 25 & $672,3 \pm 1,5$ & $0,149 \pm 0,002$ & $300,55 \pm 0,46$ \\
\hline 30 & $767,2 \pm 1,8$ & $0,179 \pm 0,002$ & $298,64 \pm 0,24$ \\
\hline 35 & $790,8 \pm 1,7$ & $0,195 \pm 0,001$ & $299,45 \pm 0,65$ \\
\hline 40 & $861,2 \pm 2,0$ & $0,199 \pm 0,002$ & $298,14 \pm 0,27$ \\
\hline
\end{tabular}

Tabla 4. Densidad, conductividad térmica y temperatura para el carbón en polvo con ACPM.

\begin{tabular}{|c|c|c|c|}
\hline$\%$ ACPM & $\left.\boldsymbol{\rho}\left(\mathbf{K g} / \mathbf{m}^{3}\right)\right)$ & $\boldsymbol{k}\left(\mathbf{W m}^{-1} \mathbf{K}^{-1}\right)$ & $\boldsymbol{T}(\mathbf{K})$ \\
\hline 0 & $677,2 \pm 2,0$ & $0,063 \pm 0,003$ & $300,09 \pm 0,06$ \\
\hline 5 & $626,3 \pm 1,3$ & $0,106 \pm 0,002$ & $301,17 \pm 0,16$ \\
\hline 10 & $632,1 \pm 1,6$ & $0,121 \pm 0,002$ & $302,65 \pm 0,08$ \\
\hline 15 & $661,4 \pm 2,0$ & $0,142 \pm 0,003$ & $301,15 \pm 0,08$ \\
\hline 20 & $733,5 \pm 1,5$ & $0,181 \pm 0,001$ & $301,31 \pm 0,09$ \\
\hline 25 & $748,0 \pm 2,2$ & $0,192 \pm 0,003$ & $289,17 \pm 0,10$ \\
\hline 30 & $791,2 \pm 1,6$ & $0,199 \pm 0,003$ & $301,14 \pm 0,12$ \\
\hline 35 & $891,5 \pm 2,2$ & $0,201 \pm 0,001$ & $299,16 \pm 0,09$ \\
\hline 40 & $935,2 \pm 2,8$ & $0,215 \pm 0,002$ & $299,64 \pm 0,09$ \\
\hline
\end{tabular}

Al analizar los valores de $\rho, k$ y $T$, para las muestras de carbón con concentraciones de fluido superiores al 5\% e inferiores al 40\% (tablas 2, 3 y 4), se observa que la densidad volumétrica tiende a aumentar con la concentración del fluido, y se aprecia que dicho parámetro oscila entre $733 \pm 3,7 \mathrm{~kg} / \mathrm{m}^{3}$ y $926 \pm 2,1 \mathrm{~kg} / \mathrm{m}^{3}$ para la mezcla con agua; entre $664 \pm 1,3 \mathrm{~kg} / \mathrm{m}^{3}$ y $861 \pm 2,0 \mathrm{~kg} / \mathrm{m}^{3}$ para la muestra con alcohol, mientras 
que cuando se varía la concentración de ACPM, la densidad varía entre $677 \pm 2,0$ $\mathrm{kg} / \mathrm{m}^{3}$ y $935 \pm 2,8 \mathrm{~kg} / \mathrm{m}^{3}$.

La conductividad térmica $(k)$ se incrementa al aumentar la concentración en peso del aglutinante agregado, para la temperatura reportada (ambiente), encontrándose que los valores experimentales de $k$ varían en un rango de $0,112 \pm 0,004) \mathrm{Wm}^{-1} \mathrm{~K}^{-1}$ a $0,277 \pm 0,003 \mathrm{Wm}^{-1} \mathrm{~K}^{-1}$, cuando se utiliza agua como fluido, mientras que para el alcohol, el rango de variación es de $0,101 \pm 0,004 \mathrm{Wm}^{-1} \mathrm{~K}^{-1}$ a $0,199 \pm 0,002 \mathrm{Wm}^{-1} \mathrm{~K}^{-1} \mathrm{y}$ para el ACPM osciló entre $0,106 \pm 0,002 \mathrm{Wm}^{-1} \mathrm{~K}^{-1}$ y $0,215 \pm 0,002 \mathrm{Wm}^{-1} \mathrm{~K}^{-1}$. De los resultados anteriores, se puede afirmar que la mezcla de carbón con agua, presenta la mayor conductividad térmica.

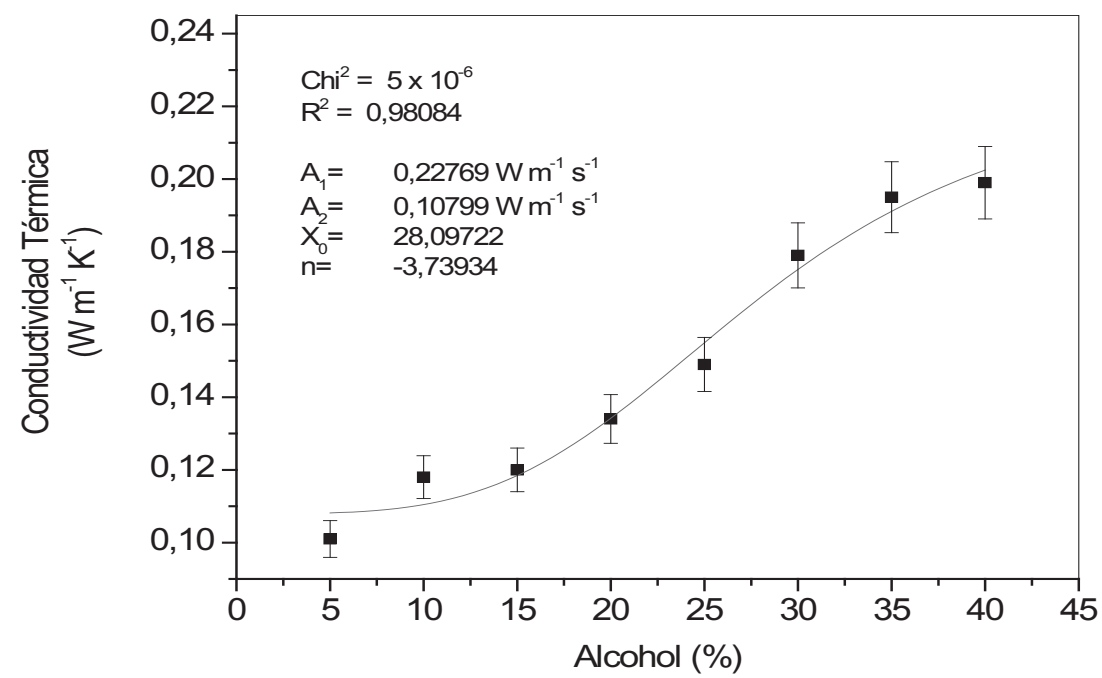

Figura 2. Conductividad térmica en función de la concentración de alcohol agregada a la muestra

El comportamiento de $\boldsymbol{k}$ en función de la concentración del aglutinante agregado (alcohol, agua y ACPM), se presenta en las figuras 2, 3 y 4 respectivamente, en donde la línea sólida corresponde al mejor ajuste a los datos experimentales, usando una función logística de crecimiento, con límite de saturación dada por la expresión (6) [16].

$$
y=\frac{\left(A_{1}-A_{2}\right)}{\left[1-\left(x / x_{o}\right)^{n}\right]}+A_{2}
$$

Donde $A_{2}$ corresponde al valor de la conductividad térmica $(y)$ de la muestra cuando se agrega una cantidad mínima de aglutinante $(x=5 \% w t) ; A_{1}$ es el valor de la propiedad térmica cuando la concentración del aglomerante es muy grande $(x>>)$, es 
decir, el valor máximo de saturación; $x_{0}$ hace referencia al punto donde se produce el cambio de curvatura, y $n$ es la razón de crecimiento. De lo anterior se aprecia que el parámetro $\mathrm{A}_{2}$ (figuras 2,3 y 4), corresponde exactamente a los datos reportados para $\boldsymbol{k}$ en las tablas 2, 3 y 4 para las concentraciones bajas del aglutinante (5\%). En estas mismas gráficas, al analizar el parámetro $A_{1}$, se observa que el valor de $\boldsymbol{k}$ para la mayor concentración de aglutinante (alcohol, agua y ACPM), reporta los valores de $0,228 \mathrm{Wm}^{-1} \mathrm{~K}^{-1}, 0,338 \mathrm{Wm}^{-1} \mathrm{~K}^{-1}$ y $0,213 \mathrm{Wm}^{-1} \mathrm{~K}^{-1}$ respectivamente. Revisando los coeficientes de correlación $\left(R^{2}\right)$, y las desviaciones cuadráticas media $\left(C^{2}{ }^{2}\right)$, en los ajustes realizados a los datos experimentales, usando la expresión (6), se observa que los valores reportados para los parámetros $A_{1}$ y $A_{2}$ son confiables, pues $R^{2}$ es muy cercano a la unidad, y $\mathrm{chi}^{2}$ es del orden de $10^{-6}$ (figuras 2,3 y 4 ).

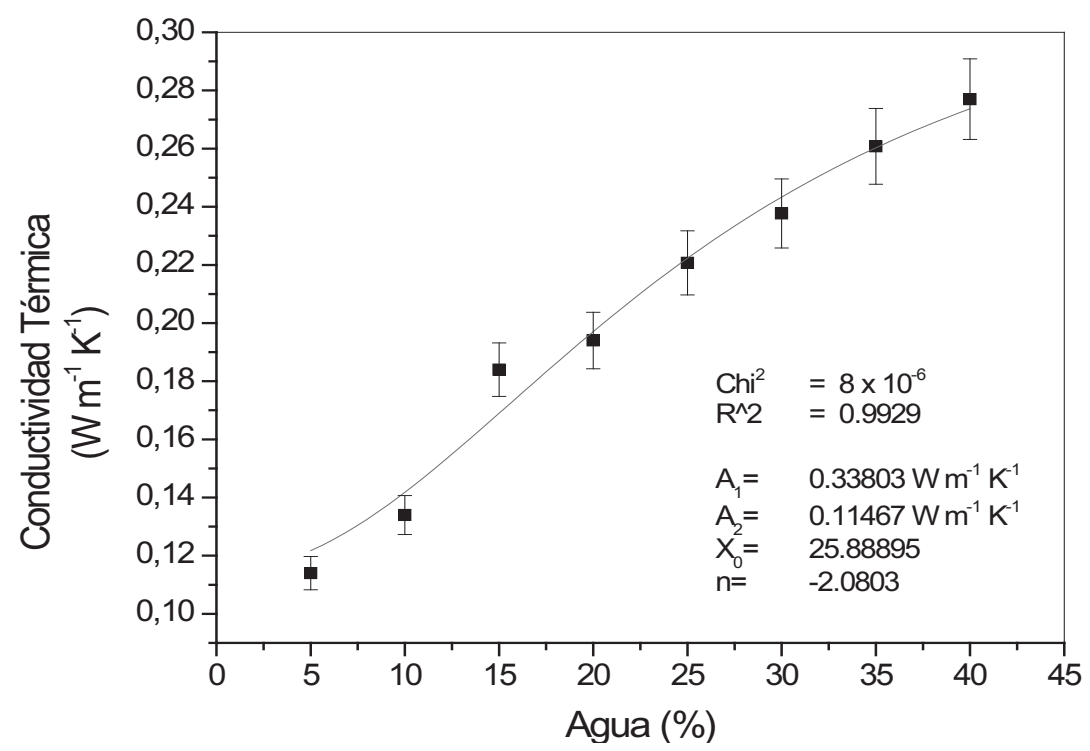

Figura 3. Conductividad térmica en función de la concentración de agua agregada a la muestra

Comparando los resultados de $\boldsymbol{k}$ a temperatura ambiente, reportados en la literatura para el polvo de carbón $\left(0,065 \mathrm{Wm}^{-1} \mathrm{~K}^{-1}\right)$ [15], para el agua $\left(0,58 \mathrm{Wm}^{-1} \mathrm{~K}^{-1}\right)$ [17], el alcohol $\left(0,17 \mathrm{Wm}^{-1} \mathrm{~K}^{-1}\right)$ [18], y el ACPM $\left(0,15 \mathrm{Wm}^{-1} \mathrm{~K}^{-1}\right)$ [17], con los hallados experimentalmente en este trabajo (tablas 2,3 y 4 ), se aprecia que están en el mismo orden de magnitud, y que los valores de $\boldsymbol{k}$ aumentan al incrementar la concentración del aglutinante (agua, alcohol y ACPM), coincidiendo con lo reportado por Raffi M., et al [19]. 


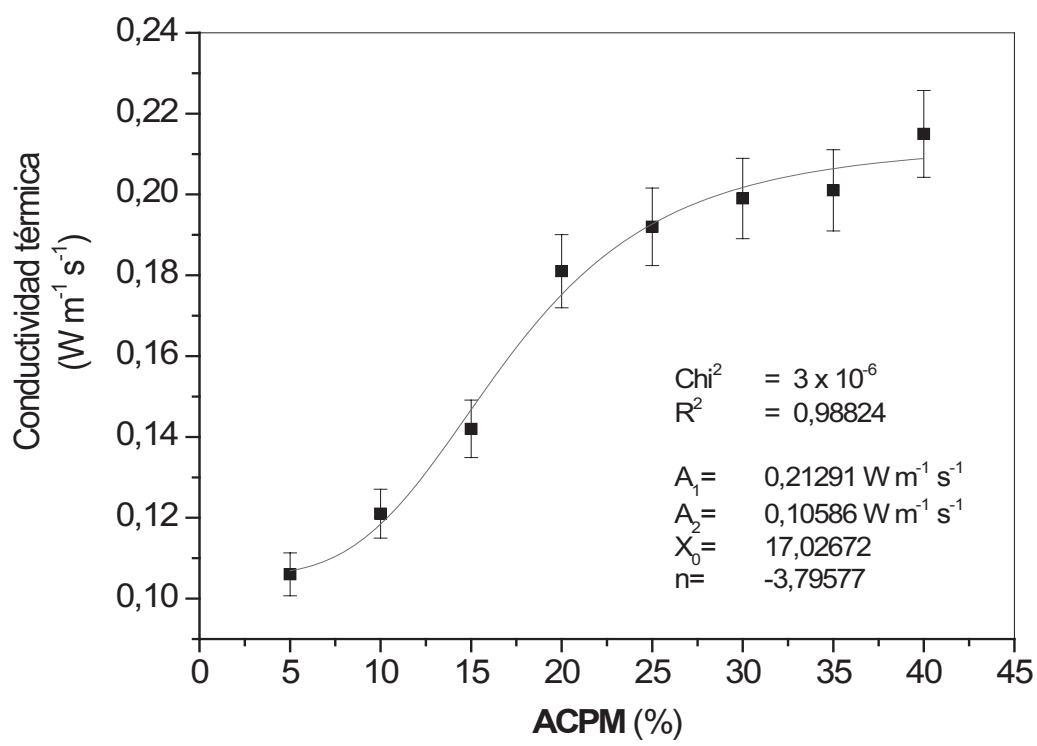

Figura 4. Conductividad térmica en función de la concentración de ACPM agregada a la muestra

\section{CONCLUSIONES}

Del análisis sobre el porcentaje de ceniza, materia volátil, carbón fijo, azufre y el poder calorífico realizado a las muestras de polvo de carbón, se concluye que el carbón de la mina Cerro Tasajero, corresponde a un carbón tipo bituminoso clasificado como alto volátil, que puede ser utilizado como carbón aglomerante.

Se reporta el valor de la conductividad térmica a temperatura ambiente, de muestras de polvo de carbón de la mina de Cerro Tasajero, con tamaño de grano inferior a $150 \mu \mathrm{m}$, sometidos a una presión de compactación de $52 \mathrm{~N}$, y se encontró un valor promedio de $0,063 \pm 0,003 \mathrm{~W} \mathrm{~m}^{-1} \mathrm{~K}^{-1}$.

El análisis de la conductividad térmica $(\boldsymbol{k})$ en función de la concentración del aglutinante (agua, alcohol y ACPM), permite concluir que al aumentar su concentración, se incrementa el valor de $\boldsymbol{k}$, lo cual fue corroborado con el comportamiento de la densidad de la muestra, que también se incrementa al aumentar la concentración de fluido agregado. El comportamiento de k en función de la concentración del aglutinante, se ajustó a una función logística de crecimiento con límite de saturación dada por la expresión (6), y se reportaron los valores de $\boldsymbol{k}$ correspondientes a la saturación de la mezcal carbón-aglutinante, dados por el parámetro $A_{1}$ presente en la función de ajuste, valores que pueden considerase confiables, por que el coeficiente de correlación $\mathrm{R}^{2}$ es muy cercano a la unidad y la desviación cuadrática media es muy pequeña del orden de $10^{-6}$. 
En general, se encontró que las muestras que presentaron la mayor conductividad térmica fueron las que utilizaron agua como aglutinante debido, muy posiblemente, a la bipolaridad de la molécula de agua.

\section{AGRADECIMIENTOS}

Al Fondo de Investigaciones Universitarias (FINU), de la Universidad Francisco de Paula Santander (UFPS), por su apoyo económico. Al Centro de Investigación en Materiales Cerámicos CIMAC-UFPS, por el préstamo de sus instalaciones y a Fredy Carrillo y Yuliet Pabón del programa Especialización en Física (UFPS) por su colaboración en la preparación de las muestras.

\section{REFERENCIAS BIBLIOGRÁFICAS}

[1] Sehlke G. Wichlacz P. (2009). U. S. Department of Energy's Role in the Energy-Water Nexus. In: Journal of Contemporary Water Research and Education, Vol. 143, pp. 56-62.

[2] Hiroaki Y., Nobuhiro M. and Makoto T. (2003). Coal Firing Power Generation Technology. In: Journal of the Japan Institute of Energy, Vol. 82, pp. 822-829.

[3] Vieira C. M. F. and Monteiro S. N. (2009). Incorporation of solids wastes in red ceramics - an update review. In: Revista Materia, Vol. 14, pp. 881-905.

[4] Santurio Díaz J. (2002). Corrección y mejora del análisis de humedad del carbón térmico por medios no destructivos en tiempo real. En: Universidad de Oviedo.

[5] Plitt L. R. (1998). Basic physical principles of on-line coal ash and moisture measurement. In: Seminar on coal moisture and ash on-line analyzers.

[6] Unsworth J. F. Barrta D. J. Roberts P. T. (1991). Coal Quality and Combustion Performance (2007). An international perspective. In: Coal Science and Technology, Vol. 19, pp. 638-643.

[7] Valbuena O. (2007). Efecto de la distribución del tamaño de partícula en la conductividad térmica de polvo de carbón usado en la elaboración del combustible tipo CCTA. En:_Universidad Francisco de Paula Santander.

[8] Flórez P. (2006). Mercado Nacional e internacional del carbón colombiano, En: Ministerio de Minas y energía, Bogotá. 
[9] Decagon D. (2006). KD2 Pro Theory. In: KD2 Pro User Manual.

[10] Carslaw, H. S., and J. C. Jaeger (1959). Conduction of Heat in Solids, $2^{\text {nd }}$ ed. Oxford, pp 258-262, London.

[11] Abramowitz M., and I. A. Stegun (1972). Handbook of mathematical functios. New York: Dover Publications, Inc.

[12] Marquard D. W., (1963). An algorithm for least-squarest estimation of nonlinear parameters, J. Soc Indust. Appl. Matb. 11: 431-441.

[13] Kluitenberg G. J., J. M. Ham, and K. L. Bristow (1993). Error analysis of the heat pulse method for measuring soil volumetric heat capacity, Soli Sci. Soc. Am. J. 57:1444-1451.

[14] Normas ASTM D: 3174, 3175, 3172, 3177 y 2015.

[15] Touloukian Y., Powell R., Ho C., and Klemens P., (1970). Thermal conductivity non metallics solids, IFI/Plenum, Vol. 2, pp. 8.

[16] Dragan A., and Scitovski R., (1996). The existence of optimal parameters of the generalized logistic function. In: Applied Mathematics and Computation, Vol. 77, pp. 281-294.

[17] Ramírez M.L.V., Nieto de Castro C. A., Nagasaka Y., Nagashima A., Assael M. J., and Wakeham W. A. (1994). Standard reference data for termal conductivity of water, AIP.ACS. pp. 1377-1381. In: http://www.nist.gov/data/PDFfiles/jpcrd493.pdf. (Consulta: mayo de 2011).

[18] Thermal Conductivity Common Liquids. In: htto://www.engineeringtoolbox.com/thermal-conductivity-liquids-d1260.html (Consulta: mayo de 2011).

[19] Raffi M. Turian, Dong-Jin Sung and Feng-Lung Hsu. (1991). Thermal conductivity of granular coals, coal-water mixtures and multi-solid/liquid suspensions. Fuel Vol. 70, Issue 10, pp: $1157-1172$. 